\title{
Diacronie
}

Studi di Storia Contemporanea

$\mathrm{N}^{\circ} 11,3$ | 2012

La satira fa storia. Eventi, pratiche, linguaggi

\section{La satira come prodotto culturale: la letteratura di cordel nella Spagna della prima metà dell'Ottocento}

Francescomaria Evangelisti e María Eugenia Gutiérrez Jiménez

\section{(2) OpenEdition \\ Journals}

Edizione digitale

URL: http://journals.openedition.org/diacronie/2688

DOI: $10.4000 /$ diacronie. 2688

ISSN: 2038-0925

Editore

Association culturelle Diacronie

Notizia bibliografica digitale

Francescomaria Evangelisti e María Eugenia Gutiérrez Jiménez, « La satira come prodotto culturale: la letteratura di cordel nella Spagna della prima metà dell'Ottocento », Diacronie [Online], № 11, 3| 2012, documento 7, Messo online il 29 octobre 2012, consultato il 02 mai 2019. URL : http:// journals.openedition.org/diacronie/2688; DOI : 10.4000/diacronie.2688 


\title{
Diacronie
}

\section{La satira come prodotto culturale: la letteratura di cordel nella Spagna della prima metà dell'Ottocento}

\author{
Francescomaria EVANGELISTI e Maria Eugenia GUTIÉRREZ JIMÉNEZ*
}

Nel XIX secolo in Spagna, parallelamente allo sviluppo della stampa liberale si continuava a produrre una stampa popolare, destinata alla lettura individuale ed alla recitazione collettiva, il cordel. Questo articolo affronta il contesto sociale, gli spazi di ricezione e consumo, le strategie di produzione e distribuzione e la ricorrenza di tòpoi in queste pubblicazioni popolari proponendo la interpretazione di alcuni elementi satirici e delle immagini.

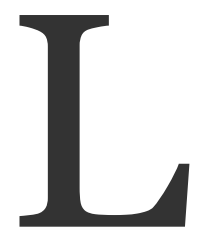

'articolo si propone di analizzare i contenuti satirici nei $\operatorname{cordel}^{1}$ pubblicati in Spagna nella prima metà dell'Ottocento.

Questa categoria di pubblicazioni popolari prende il nome dal cordel, la cordicella con cui erano appese per essere esposte, prima nelle bancarelle dei venditori ambulanti, poi, con lo sviluppo del mercato editoriale, nelle vetrine delle tipografie e delle librerie.

Essi nacquero insieme allo sviluppo della stampa e rappresentarono uno dei primi esempi di "diversificazione"editoriale. La produzione di libri e sermoni comprendeva

\footnotetext{
${ }^{1}$ Tutta la documentazione proviene dalle scatole 104 e 110 del Fondo Hazañas conservato presso la Biblioteca dell'Università di Siviglia. Questo fondo, che porta il nome del suo collezionista Joaquín Hazañas y La Rúa, bibliofilo, storico, cattedratico di Lettere e Filosofia, critico letterario e due volte rettore dell'Università di Siviglia (la Hispalense), è composto da circa novemila volumi, una copiosa edizione di fogli sciolti, relazioni di avvenimenti, canzoni popolari e romanzi volgari. Joaquín Hazañas y La Rúa realizzò quest'opera, secondo quanto racconta Casas Delgado, «influenzato da autori come Antonio Machado Álvarez, più conosciuto per il suo pseudonimo Demofilo, o il filosofo Federico de Castro Fernández, entrambi interessati nella conservazione della cultura popolare andalusa». CASAS DELGADO, Inmaculada, El éxito de lo marginal. Historia de la prensa popular en España (1750-1850). Localización, catalogación y análisis del discurso, Elaborato finale di Master, Universidad de Sevilla, 2011, p. 11.
} 
alti costi e lo stampatore, per aumentare i profitti, iniziò a sfruttare i fogli rimasti dalla stampa dei libri per creare altri prodotti, come testi di canzoni e preghiere, racconti e relazioni di avvenimenti.

La loro produzione e vendita era affidata soprattutto ai non vedenti che fin dall' inizio dell'età moderna si erano occupati di recitare, diffondendoli, i testi dei pliegos sueltos (fogli sciolti), ma la Real Orden nel $1836^{2}$ abolì i privilegi delle loro confraternite e liberalizzò il mercato.

Questi stampati, il cui prezzo $^{3}$ era simile a quello di un quotidiano attuale, si compravano per unità, senza intenzioni di collezionismo, e la loro lettura era sia individuale che condivisa in famiglia, nelle sale di lettura e nelle taverne.

I cordel erano testi di vario genere, spesso scritti in alessandrini, che raccontavano storie adatte a essere lette, recitate e ascoltate da tutti, anche da chi non sapeva leggere.

Il carattere ibrido era la loro principale peculiarità, infatti, Botrel li definiva «senza frontiere letterarie o nazionali»4, suggerendo di analizzarli in base alla combinazione di due mondi: «quello della cultura scritta e stampata con riferimento al libro e all'immagine e quello della cultura orale 5 folkloristica» ${ }^{6}$.

Per quanto questo sia un intragenere complesso, è forse possibile valutare la misura in cui il tono satirico, spesso evocato fin dal titolo, abbia influenzato la costruzione d'immaginari collettivi'.

Per comprendere il valore del satirico nella Spagna del XIX secolo, bisogna partire da un presupposto: quando si parla della satira ${ }^{8}$, si fa riferimento a una modalità ${ }^{9}$ di

\footnotetext{
2 La Real Orden del 1836 era una misura che voleva liberalizzare il mercato informativo. Con essa si decretava la dissoluzione della Confraternita della Nuestra Señora de la Visitación, opera pia dei ciechi di Madrid, e si abolivano i privilegi di cui godeva questa corporazione nella produzione, vendita e diffusione delle pubblicazioni di cordel. Con questa disposizione si stabiliva che «qualsiasi individuo che con 17 anni compiuti sappia leggere e scrivere è idoneo per questa occupazione, sommettendosi al pagamento del sussidio di commercio».

3 Il prezzo medio era di un maravadì.

4 BOTREL, Jean-François, «Palabras para el pueblo. Aproximación general a la literatura de cordel», in DIAZ GONZALEZ VIANA, Luis, Palabras para el pueblo, Vol. I, Aproximación general a la Literatura de Cordel, Madrid, Departamento de Antropología de España y América, Consejo Superior de Investigaciones Científicas, 2001, p. 42.

5 Come ha spiegato Díaz G. Viana, non va, infatti, dimenticata la sua dimensione performativa, perché questo genere complesso è costituito da storie in versi, prodotte e vendute per essere lette ma anche ascoltate e ricordate da chi non sapeva leggere. Ibidem, p. 27.

${ }^{6}$ Ibidem, p. 44.

7 Cfr. CASTORIADIS, Cornelius, The Imaginary Institution of Society, Cambridge, MIT Press, 1998 e TAYLOR, Charles, Modern Social Imaginaries, Durham-London, Duke University Press, 2004.

8 Secondo alcuni autori la satira si delinea come modalità letteraria nell'antica Roma dopo essersi già manifestata in Grecia con Archiloco e Ipponatte e sopravvive alla caduta dell'impero fino ad oggi evolvendo al pari della società per tecniche e linguaggi.

9 I moderni teorici della satira concordano unanimemente nell'intendere la satira come una modalità per la moltitudine di forme in cui si manifesta e per il suo carattere moralizzatore, per
} 
esercitare la critica da parte di un soggetto storico in un contesto sociale, tenendo presente che la socializzazione della satira, come quella del riso e dell'umorismo, non è sempre uguale in differenti società storiche.

Essa cerca di svelare aspetti taciuti dal discorso ufficiale attraverso l'uso dell'umorismo, dell'ironia e di molteplici figure retoriche; si può quindi intendere come un atto d'opposizione, di dissenso, tipico delle classi subalterne, che si scaglia contro la classe egemonica e la sua costruzione della realtà, indipendentemente dal fatto che la sua intenzione sia quella di difendere i valori tradizionali o promuovere un cambiamento.

I testi satirici offrono una rappresentazione deformata della realtà che contraddice la descrizione fornita da chi è al potere, ridicolizzando, muovendosi tra il normale, l'istituzionalizzato e tutto quello che esce dalla norma. Va quindi considerata, come indica Goodland ${ }^{10}$, la forma in cui interviene nella configurazione dei ruoli, delle identità e delle coscienze del suo pubblico ${ }^{11}$, così come nella trasformazione degli habitus ${ }^{12}$.

Per ricostruire la previsualizzazione di quello che Eco definisce il lettore modello ${ }^{13}$, occorre quindi seguire le orme lasciate nei cordel dal loro "promotore", figura che in alcune occasioni concentrava il ruolo di autore, editore e stampatore. Questo implica, oltre che descrivere il processo attraverso il quale i lettori si appropriavano del contenuto, analizzare, su base indiziaria, i suoi luoghi di ricezione, le strategie di produzione, la struttura dello stampato, il linguaggio usato, i luoghi comuni ai quali si ricorreva e il genere d'avvenimento narrato.

Si può ipotizzare che il discorso alternativo proposto nei cordel si sia diffuso in un sistema marginale di trasmissione letteraria e antitetico a quello istituzionale delle pubblicazioni liberali, che spesso erano prodotte nelle stesse tipografie.

esempio Edward e Lillian Bloom parlano di satira come modalità: «Satire suggests modality, a state of mind or feeling, a critical outlook on some detail or quality of existence» (BLOOM, Edward A., BLOOM, Lillian D., Satire's Persuasive Voice, Ithaca-London, Cornell University Press, 1979, p. 36.

${ }^{10}$ Cit. in BORDERÍA ORTIZ, Enrique, MARTÍNEZ GALLEGO, Francesc, GÓMEZ MOMPART, Josep (dirs.), La risa periodística. Teoría, metodología e investigación en comunicación satírica, Valencia, Tirant lo Blanch, 2010, p. 39. Questo libro si occupa della storia delle distinte forme della satira, nella Spagna del XIX e XX secolo, intesa come un discorso costruito culturalmente, tenendo in conto le modalità con cui il popolo può accedere alle strutture del potere e, quindi, al sapere.

${ }^{11}$ Burke invita a non dimenticare la teatralità che avvolge ogni rappresentazione, le forme con cui si rappresentano i diversi modi di stare al mondo, i simboli dello status quo o determinate attitudini morali. BURKE, Peter, Visto y no visto. El uso de la imagen como documento histórico, Barcelona, Crítica, 2001.

${ }^{12}$ Cfr. BOURDIEU, Pierre, La distinzione. Critica sociale del gusto, Bologna, Il Mulino, 1983.

13 ECO, Umberto, Lector in fabula, Milano, Bompiani, 1985. 
Il presente articolo, studiando la produzione del discorso satirico in relazione alle strutture sociali e le istituzioni politiche, si colloca in linea con la storia culturale iniziata da Roger Chartier che proponeva di superare i limiti della storia sociale della cultura e del concetto di mentalità, per approdare alla storia culturale del sociale ed al concetto di rappresentazione ${ }^{14}$.

Dato che una società, secondo la storia culturale del sociale, si può conoscere solamente attraverso le rappresentazioni ${ }^{15}$ del presente che l'uomo ha realizzato, riteniamo che, individuando il ruolo svolto dalla satira e conoscendo le pratiche sociali legate ai cordel, si possano far emergere le relazioni e le forme sociali di quel tempo.

\section{Il cordel nel XIX secolo}

La letteratura di cordel del XIX secolo manifesta una continuità nei temi e nelle forme con le pubblicazioni burlesco-satiriche del secolo precedente, anche se con delle migliorie tipografiche dovute alle innovazioni tecnologiche.

Nella parte superiore della prima pagina c'era un'illustrazione che agiva da elemento d'ancoraggio, suggerendo al lettore il contenuto e aiutando anche chi doveva mettere in scena la storia narrata.

Il titolo, che comprendeva quello che nella stampa contemporanea è comunemente definito sottotitolo, esponeva brevemente e in maniera accattivante il contenuto ma,

mentre nel XVIII secolo includeva spesso gli aggettivi "breve" e "vera", nel XIX li sostituì, seguendo il gusto del tempo, con "satirica", "burlesca" e "giocoso-seria", diventando così anche una reclame per il loro acquisto.

Dopo il corpo del testo era presente un colofone, in cui a volte si comunicava dove sarebbe stato possibile acquistare altri cordel.

L'uso di un formato standard ci suggerisce che, a differenza dei periodi precedenti, la satira godeva di una legittimità che la faceva considerare e consumare come un medium alternativo alle pubblicazioni liberali, infatti i cordel erano pensati per essere fruiti in spazi dedicati all'ozio urbano (specialmente taverne ${ }^{16}$ ), un fattore che implica,

14 CHARTIER, Roger, El mundo como representación: estudios sobre historia cultural, Barcelona, Gedisa, 1992.

15 Questo concetto è anche oggetto delle riflessioni di Carlo Ginzburg nell'articolo «Représentation: le mot, l'idée, la chose», in Annales. Économies, Sociétés, Civilisations, 6, 1991. pp. 1219-1234.

${ }^{16}$ L'incipit della Relazione giocosa della zucca e del vino: composta da un ingegno che si agitava evoca il clima in cui venivano recitati questi testi: «silenzio, attenzione, non parlate,/ ascoltate compagni,/ vi racconterò un caso,/ che mi è successo a Granada/ però affinché io lo racconti/ dovete stare con le mani incrociate/ con silenzio ed attenzione,/ senza sbattere le 
come nel caso dei manifesti, dei giornali o degli spettacoli ottici (i diorami), la possibilità di utilizzo sia diretto che mediato, sia collettivo che individuale.

Neanche la funzione sociale della satira era più la stessa: Bajitin sostiene che nel romanticismo si sia prodotta una «risurrezione del grottesco [...] ma non con il senso che la risata del realismo grottesco [...] ebbe per le classi popolari del Medio Evo»17, infatti la letteratura di cordel del XIX secolo, pur essendo ancora un prodotto della manifattura culturale, riscuoteva successo anche e soprattutto nelle classi medio alte ${ }^{18}$, tra le quali si stava diffondendo il costrumbrismo ${ }^{19}$.

Le opere di Goya Baile a orillas del río Manzanares (1777, Madrid), La vendimia (1786-87, Madrid) o La gallina ciega (1788-89, Madrid) ci mostrano come molti degli appartenenti alla classe intermedia e alcuni aristocratici "imborghesiti”, avessero scelto di identificarsi con le tradizioni del volgo. Caro Baroja aggiunge che in questo periodo si verificò «un gioco di relazioni nell'ordine estetico che fa sì che le classi sociali, rigidamente stabilite nell'antico regime, solidarizzino per quelle inferiori: per il "popolare" e per quello che è quasi più in basso del popolare, ossia la gitanesca, la jacarandina y el hampa»20.

La borghesia continuava ad avere poca capacità reale di partecipare alla vita politica del Paese e di diventare la classe dominante, ma aveva sviluppato un nuovo strumento per influenzare la neonata opinione pubblica e raggiungere il potere: il giornalismo.

ciglia/ senza tossire, senza sputare/ senza colpire la cassa [suonare le percussioni su una cassa di legno]/ senza suonarsi le narici».

17 BAJTIN, Mijail, La cultura popular en la Edad Media y en el Renacimiento. El contexto de François Rabelais, Madrid, Alianza, p. 39.

${ }^{18}$ In Spagna il passaggio da una società feudale a una di classi non seguì un cammino simile a quello di altre realtà occidentali, ma presentò numerose contraddizioni. La rivoluzione politica scaturita dalla Guerra di Indipendenza (1808-14) non fu accompagnata da una rivoluzione dei rapporti economici. Nella prima metà del XIX secolo, con la restaurazione di Fernando VII, i liberali avevano dovuto scegliere l'esilio, lasciando il Paese privo di una borghesia consolidata e quindi senza uno sviluppo del commercio tale da favorire la rivoluzione industriale. Lo smantellamento del tradizionale sistema economico spagnolo, iniziato con il "decennio nefasto spagnolo" di Fernando VII, continuò durante l'epoca di Maria Cristina (1833-40) e il regno di Isabella II (1844-75). Con la morte nel 1833 di Fernando VII e con l'approvazione nel 1837 della Costituzione, nell'ambito politico avvenne un lieve rinascimento del liberalismo, propiziato dal ritorno degli esiliati e emigrati, come conseguenza dell'amnistia e della politica conciliatrice di Martinez de la Rosa.

19 Il costumbrismo è un genere artistico che si sviluppò in Spagna nel XIX secolo, basato sulla realizzazione di opere ispirate alle tradizioni popolari. In un'epoca di stravolgimenti sociali si credeva che solo l'essenza "del popolo" fosse rimasta genuina. Ramón de Mesonero Romanos e Mariano José de Larra furono i suoi principali interpreti in campo letterario.

${ }^{20}$ CARO BAROJA, Julio, Ensayo sobre la Literatura de Cordel, Madrid, ISTMO, 1990, p. 25. Con gitanesca si intendono le composizioni letterarie che si ispiravano ai gitani; la jacarandina era un genere satirico che, usando il gergo criminale, narrava episodi di questo mondo esercendo una critica sociale (tra gli autori più rappresentativi vi erano Calderón de la Barca e Francisco de Quevedo); infine, col termine hampa si definivano le composizioni che si ispiravano alle gesta di picari e delinquenti. 
Bozal afferma che «la borghesia liberale si serve della stampa per emanare la propria ideologia: critica alacremente l'antico regime e i suoi rappresentanti innalzando la bandiera del razionalismo e del progresso, e fissa lentamente i suoi archetipi sociali»21. Quindi essa non va solo intesa come una classe intermedia ma anche come un gruppo sociale che stava configurando la propria identità e che, per questo, subì anche l'influsso delle letterature emarginate, care alle classi più popolari, che tanto ispiravano le mode borghesi.

Lo sviluppo del liberalismo borghese spagnolo fu accompagnato dalla promulgazione di leggi che regolavano la libertà di stampa e dall'instaurazione di un sistema liberale di controllo editoriale. Con il Decreto Reale del 1834, approvato nello stesso anno dell'abolizione del Tribunale della Santa Inquisizione, si stabiliva che sarebbero rimasti liberi dall'obbligo di censura preventiva quei periodici «puramente tecnici o [che] trattassero solo le arti, le scienze naturali o la letteratura».

Con lo scopo di aggirare le misure repressive, nacque allora una stampa minore basata su "semi-periodici", volantini e opuscoli, di carattere sedizioso e senza un'apparente periodicità, anche se in realtà «la periodicità si compiva fino a rendere possibile le sottoscrizioni» ${ }^{22}$.

Le istituzioni provarono a limitare queste iniziative definendo, con la legge del 22 marzo 1837, cosa dovesse essere considerato "periodico" ma, paradossalmente, fu proprio la periodicità a non essere considerata una caratteristica essenziale.

Con il Decreto del 9 aprile del 1844 si stabilirono le sanzioni per la produzione e distribuzione di opuscoli e fogli volanti non autorizzati ma nella cornice normativa, il cordel sfuggiva alle categorie stabilite e si collocava in un vuoto legale che ne permetteva la sopravvivenza: tale indeterminatezza spinge a chiedersi se le autorità, non menzionando il cordel nelle norme, avessero preferito un tacito lassismo.

Proprio per questo, non sorprende che a tali pubblicazioni si siano opposti gli agenti della stampa liberale che, secondo Pura Fernández, «invocano il controllo fiscale e della polizia e cominciano ad appoggiare la creazione di gruppi di pressione come L'apostolato della Stampa o l'Associazione Padri di Famiglia, che si ergono a guardiani della pubblica morale» ${ }^{23}$.

\footnotetext{
${ }^{21}$ BOZAL, Valeriano, «La ilustración gráfica del siglo XIX en España», Madrid, Alberto Corazón, 1979, cit. in LLERA, Josè Antonio, «Una historia abreviada de la prensa satírica en España: Desde El Duende Crítico de Madrid hasta Gedeón», in Estudios sobre el Mensaje Periodístico, 9/2003, p. 206.

${ }_{22}$ ELORZA, Antonio, «Un vacío legal: periódicos y hojas volantes republicanos (1840-1843)», in Estudios de Información, 23, 1972, pp. 51-63, cit in DİAZ G. VIANA, op. cit., p. 74.

${ }_{23}$ Cit. in DÍAZ G. VIANA, op. cit., p. 97.
} 
Secondo l'autrice, nel fuoco incrociato tra conservatori, moralisti cattolici e difensori delle libertà politiche e d'espressione sorse un nemico comune, la letteratura di cordel diffusa dai ciechi che, nonostante gli sforzi dei suoi nemici, avrebbe dimostrato di possedere «una natura incombustibile, e non saranno la legislazione né la censura morale, le armi chiamate per sconfiggerla» 24 .

\section{La letteratura di cordel ai margini della storia}

La poesia popolare, uno dei principali generi della letteratura di cordel, che trovava nel lessico giocoso un veicolo perfetto per comunicare il senso tragicomico della vita, era discriminata sia da molti intellettuali coevi, per la presunta e intrinseca immoralità, che corrompeva lo spirito del popolo, sia per i guardiani del decoro. Il vincolo con il gusto "borghese" e la forte identità "popolare", respinti dalla vigente concezione aristocratica della cultura, furono quindi la causa della sua condizione marginale negli studi letterari e storiografici successivi.

Questo sembra confermato dal fatto che Duran, autore del Romancero general o colección de romances castellanos anteriores al siglo XVIII, criticò duramente questo tipo di letteratura, definendone il contenuto come un «pantano di corruzione, di falsa scienza e di fede persa» ed escludendola dalla sua antologia.

Fino agli anni Settanta dello scorso secolo, la condizione di "genere minore" ha determinato una scarsa attenzione storiografica verso questi fenomeni, con la conseguenza che sono stati fatti pochi tentativi di ricostruire il loro ruolo nella trasmissione di quello che adesso definiamo cultura popolare.

Nell'ambito andaluso possiamo trovare pochi testi dedicati alle forme con cui la satira si presenta nella stampa. Sono degne di menzione le opere di Miguel Ángel Gamonal, La ilustración gráfica y la caricatura en la prensa granadina del siglo XIX ${ }^{25}$ o lo studio di Fernando Arcas Cubero, El País de la Olla. La imagen de España en la prensa satírica malagueña de la restauración ${ }^{26}$, dove è evidente la volontà dell'autore di definire una cornice semiologica che aiuti nell'interpretazione delle immagini satiriche.

\footnotetext{
24 Ibidem.

25 GAMONAL, Miguel Ángel, La ilustración gráfica y la caricatura en la prensa granadina del siglo XIX, Granada, Diputación Provincial, 1983.

${ }_{26}$ ARCAS CUBERO Fernando, El País de la Olla. La imagen de España en la prensa satírica malagueña de la restauración, Malaga, Arguval, 1990.
} 
La "discriminazione" storiografica è forse stata causata dal fatto che la stampa satirica sia sempre stata considerata come una stampa minore, basata su un linguaggio "basso" e violento, partorita e consumata da elementi marginali della società, per raccontare tutto quello che è situato tra il normalmente accettato e il censurato.

\section{Analisi dei racconti burlesco-satirici}

Iniziamo la nostra analisi da uno degli argomenti più usati dagli autori di satira, la contrapposizione tra ricchi e poveri, dove questi ultimi spesso abusano della sprovvedutezza dei "signorini” di città.

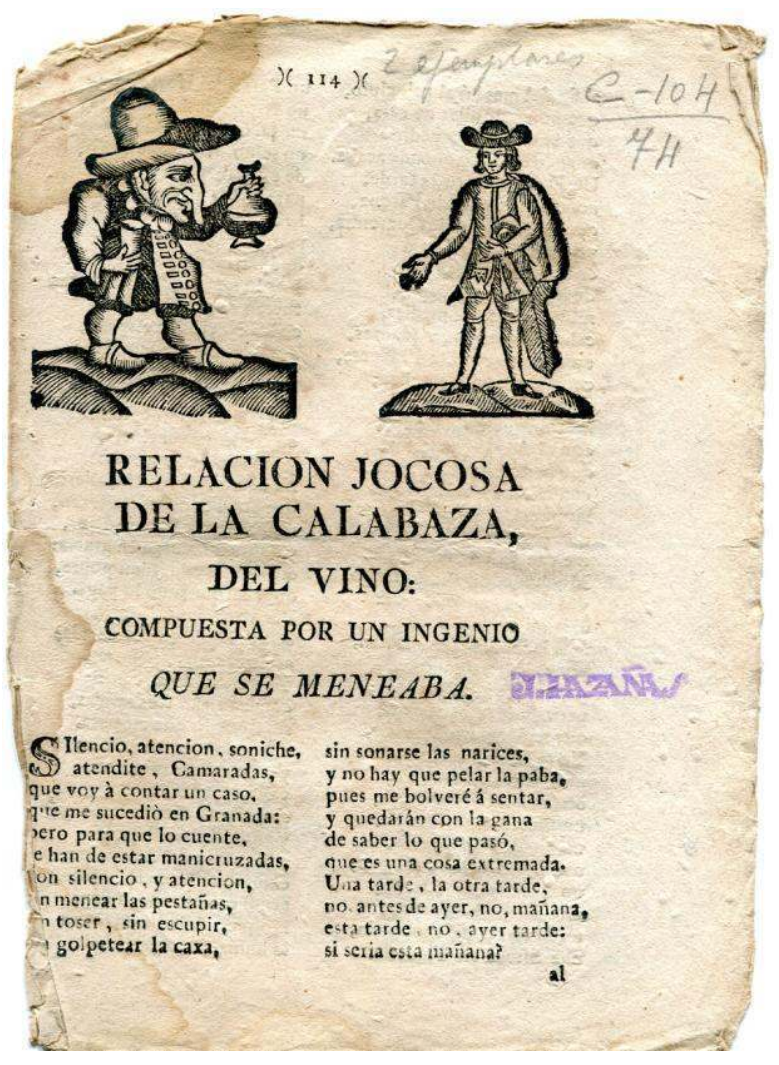

Nella Relazione giocosa della zucca e del vino: composta da un ingegno che si agitava ${ }^{27}$, viene trattata in maniera satirica la disputa sulla comparazione tra il modo di vivere del ricco rispetto a quella del povero, criticando le mode esterofile dei damerini di città ed il loro stile di vita. Il protagonista è un giovane ben vestito che passeggia nei dintorni dell'Alhambra e che viene raggirato da un furbo popolano. L'autore ha usato la prima persona per raccontare l'aneddoto, come se vi avesse partecipato realmente e nell'illustrazione è rappresentato con le caratteristiche iconografiche del satiro-buffone mentre il giovane appare come un dandy ridicolo.

Già affrontato dalla letteratura canonica nella figura dello scudiero Lazarillo de Tormes o nell'hidalgo del El Alcalde de Zalamea, questo topos viene sviluppato nella Relazione giocoso-seria dei trenta reali ${ }^{28}$, ricreando quanto accaduto ad un "povero damerino con una signora marchesa de Boardilla» che dopo averlo fermato per la strada ed avergli elencato le sue illustri parentele per abbindolarlo, gli chiede trenta

${ }^{27}$ La trascrizione dei titoli verrà mantenuta come in originale: RELACION JOCOSA DE LA CALABAZA, DEL VINO: COMPUESTA POR UN INGENIO QUE SE MENEABA.

${ }^{28}$ RELACION JOCO-SERIA DE LOS TREINTA REALES. Senza dati di edizione. 
denari. In quest'occasione, come possiamo osservare nell'illustrazione, la storia sembra avvenire sotto l'attento sguardo di un satiro deforme, per cui è evidente che era abituale ridere, non solo delle disavventure narrate, ma anche delle disgrazie altrui.

Questo cinismo narrativo sembra eccitare il popolo, forse «guidato da un sentimento subcosciente della propria frustrazione, unito ad un desiderio cosciente di scherzare ${ }^{29}$, cosa evidente in un altro racconto, che ha come protagonista un "pover'uomo", Marcos Cabra, caduto in disgrazia dopo essersi sposato.

$\mathrm{Si}$ tratta della Giocosa relazione, nella quale si racconta il tragico sposalizio di un giovane disgraziato, [...] che dopo di un allegro matrimonio, sperimentò in pochi mesi così tanti parti nella propria casa, che per assistervi

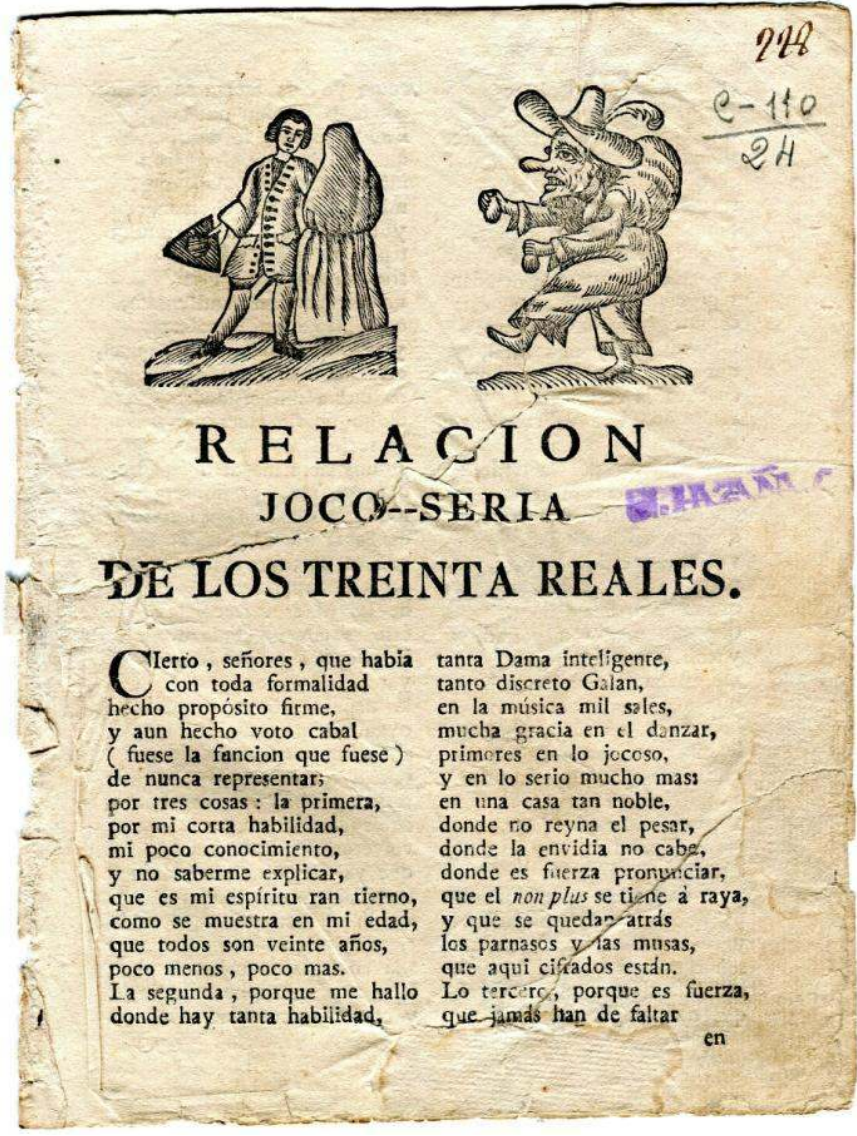
non ha potuto mangiare né riposare per tutto il giorno. Rendetevi conto della sua grande afflizione e delle molte altre circostanze che vedrà colui che non sia cieco ${ }^{30}$. Il cordel si conclude con un monito per il lettore: «e così, amici, se vi sposate/ cercate una donna che non partorisca/ e non avrete altra cosa/ che voi in casa.»

Questo testo evoca un altro topos ricorrente nella satira di cordel: le donne che spesso rappresentano la causa delle disavventure vissute dai protagonisti. Un esempio è la Satira graziosa dei quindici fidanzati. Nella quale si manifestano tutti i vezzi, le mode e le lusinghe spese dalle signorine donzelle quando vedono che hanno molti

29 CARO BAROJA, Julio, op. cit., p. 205.

30 JOCOSA RELACION, EN QUE SE REFIERE EL trágico casamiento de un desgraciado Mozo, llamado MARCOS DE CABRA, vecino de la Ciudad de Guadarrama, que despues de unas alegres bodas, experimentó à pocos meses tanta multitud de partos en su casa, que por asistir à ellos, no pudo comer ni descansar en todo el dia. Dase cuenta de su gran afliccion, y de otras muchas circunstancias que verá el que no fuere ciego. Senza dati di edizione. 
fidanzati e che questi muoiono per il loro amore, con gli altri che potrà vedere il curioso lettore ${ }^{3}$.

Questa satira fu illustrata con un'immagine che poteva evocare nel lettoreascoltatore alcuni dei Capricci di Goya (1799), soprattutto le incisioni che ridicolizzavano i rapporti tra i due sessi. Nel descrivere le professioni dei vari fidanzati, inoltre, l'autore ne traccia un'immagine poco lusinghiera, forse attinta dalla vulgata, come nel caso del veterinario che prolunga la cura secondo il suo compenso, del sarto che va vestito con gli scampoli sottratti ai clienti o del ristoratore che espleta le sue funzioni fisiologiche nella zuppa.

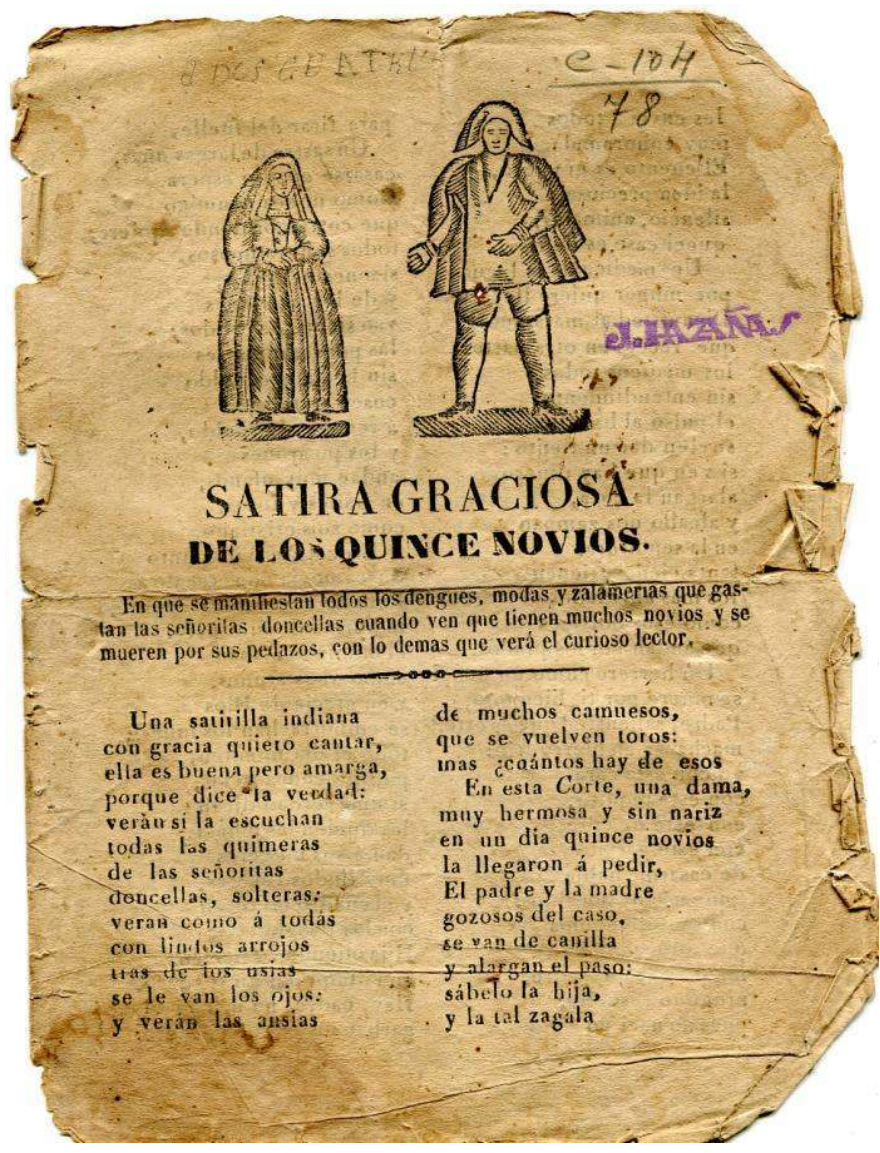

L'uso dell'immagine e la descrizione delle professioni ci suggeriscono che il potere della satira non si esauriva nella ridicolizzazione ma tendeva a riprodurre archetipi, che già erano nell'immaginario collettivo, per guidare il pubblico alla costruzione del senso della storia. Nel racconto della vita coniugale, si può osservare il manifestarsi di un antifemminismo ${ }^{2}$ che, in questo tipo di satira, non risparmiava nessuna donna, sia che fosse sposata, nubile, anziana o giovane.

Dagli strali degli autori di cordel non sembrava salvarsi nemmeno gli uomini, anche se ne escono rappresentati in modo più benigno delle donne, poiché dipinti come vittime degli abusi perpetrati da queste ultime.

${ }^{31}$ SATIRA GRACIOSA DE LOS QUINCE NOVIOS. En que se manifestan todos los dengues, modas y zalamerias que gastan las señoritas doncellas cuando ven que tienen muchos novios $y$ se mueren por sus pedazos, con lo demas que verá el curioso lector.

${ }^{32}$ Caro Baroja catalogò un altra edizione, risalente al secolo precedente con un lieve cambio nel titolo, visto che i fidanzati in questo caso erano undici: CARO BAROJA, Julio, op. cit., p. 201. 
I contenuti delle satire sembrano voler offrire delle ragioni per fuggire dal matrimonio, come nella Relazione di un giovane celibe, manifestando i motivi per non sposarsi33 e nel Divertente resoconto di un feroce litigio che hanno avuto un vecchio ed una vecchia di cento e ottanta anni, per la gelosia di lei, ed i particolari e divertenti inganni che avvennero tra i due, come la giustizia li condusse in prigione per fare amicizia, con altri avvenimenti speciali34.

In un'altra categoria troviamo i racconti ambientati nel mondo criminale, che facevano leva sul codice d'onore che animava e anima questo settore della società. Un esempio può essere il Racconto grazioso e burlesco, per far ridere e passare il tempo a chi ha la pancia ben piena, riguardo ad uno spione, chiamato Sancho Cornillo, e quello che gli accadde 35 .

In questo caso il divertimento per l'ascoltatore/lettore era rappresentato dal castigo ricevuto

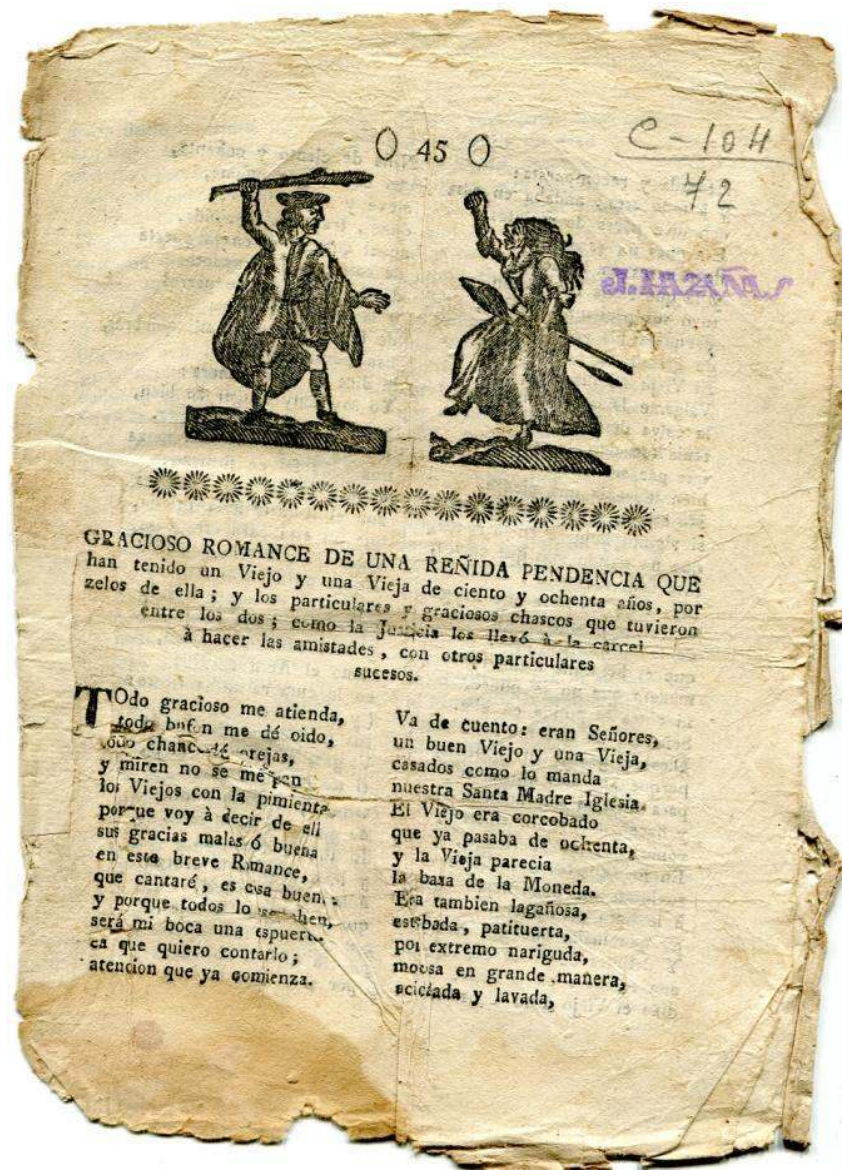
dal protagonista per essere un informatore della polizia e conteneva un monito a non collaborare con le autorità.

33 RELACION DE UN MOZO SOLTERO, MANI-festando los motivos para no casarse. Reca nel colofone: «Con licenza: In Cordova, nella stamperia di Don Rafael Garcia Rodríguez».

34 GRACIOSO ROMANCE DE UNA REÑIDA PENDENCIA QUE han tenido un Viejo y una Vieja de ciento y ochenta años, por zelos de ella; y los particulares y graciosos chascos que tuvieron entre los dos; como la Justicia los llevó á la carcel á hacer las amistades, con otros particulares sucesos. Senza dati di edizione.

35 ROMANCE GRACIOSO, Y BURLESCO, PARA reir y pasar tiempo el que tuviere la barriga bien llena, por un soplon, llamado SANCHO CORNILLO, y lo que sucediò. Il colofone reca: «Con licencia: En Córdoba, en la Imprenta de Doña “...ria” [errata] de Ramos, y Coria Plazuela las Cañas». 


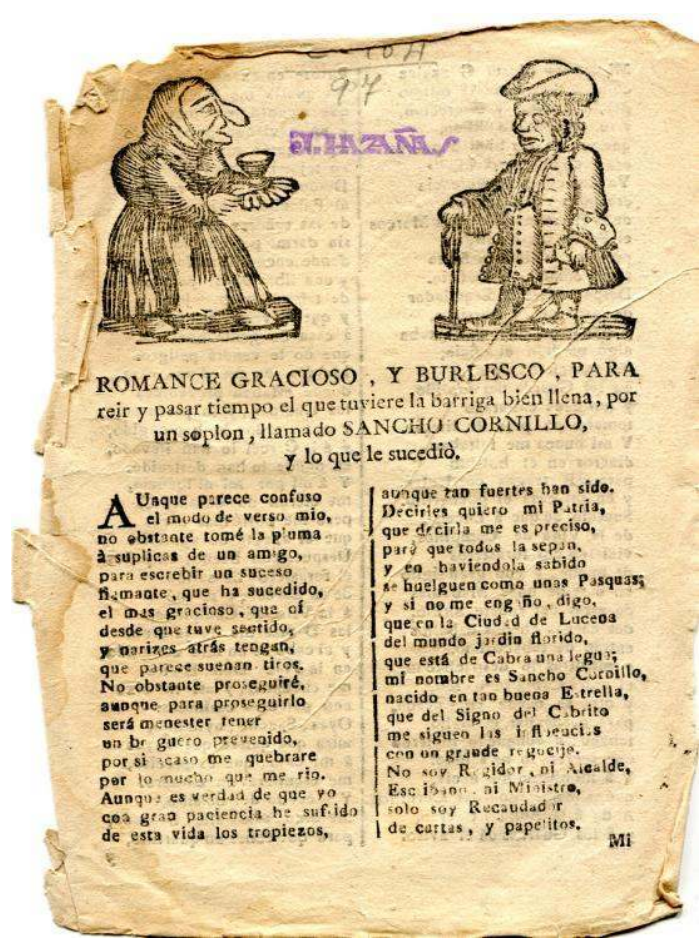

Un altro genere di satira si trova nell'Opera molto divertente per ridere e passare il tempo, che si chiama il Testamento del Gallo. È scritta con uno stile molto curioso: $e$ alla fine dell'opera ci sono alcune bravate e le lacrime di un Ruffiano, lungo di lingua e corto di mani. Composta da Crisaoval Bravo, Cieco, nativo di Cordova ${ }^{36}$.

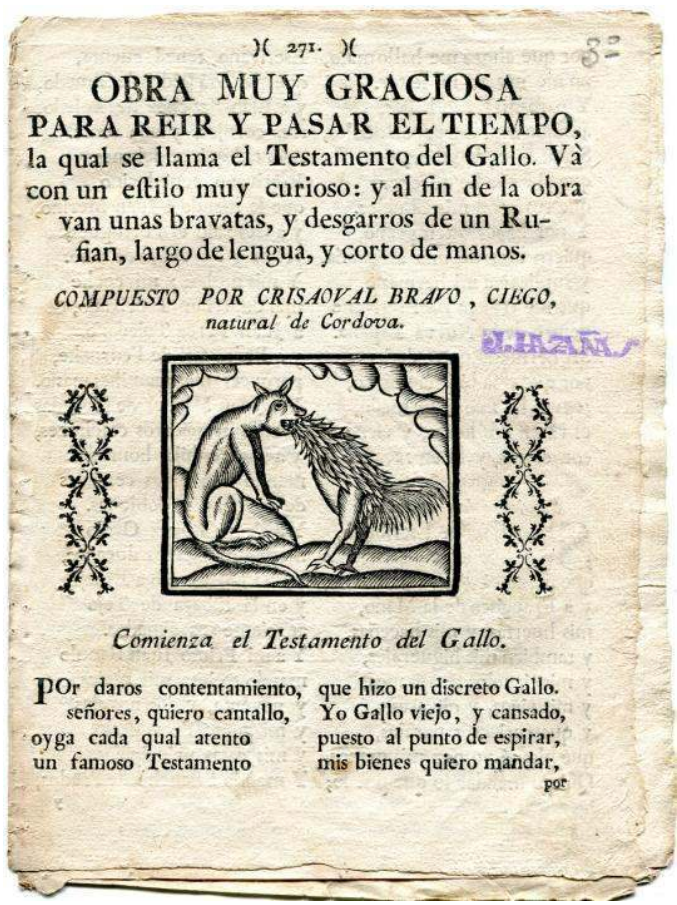

${ }^{36}$ OBRA MUY GRACIOSA PARA REIR Y PASAR EL TIEMPO, la qual se llama el Testamento del Gallo. Và con un estilo muy curioso: y al fin de la obra van unas bravatas, y desgarros de un Rufian, largo de lengua, y corto de manos. COMPUESTO POR CRISAOVAL BRAVO, CIEGO, natural de Cordova. Senza dati di edizione. 
In questo caso, come si può osservare nell'illustrazione, il protagonista subisce un processo di animalizzazione che evidenzia i lati più censurabili della sua condotta.

In altre pubblicazioni dello stesso tenore non si condannava un'attitudine individuale ma le storiche contrapposizioni legate alla parentela, come nel racconto Curiosa e generale battaglia che ordinariamente si verifica tra Suoceri e Generi, Suocere e Nuore, la cui comune sventura è da tutti posseduta e da nessuno desiderata. Si riferiscono in questa le condizioni, le proprietà e la delusione della milizia dei suoceri e degli avviliti generi37. L'autore di questo testo includeva

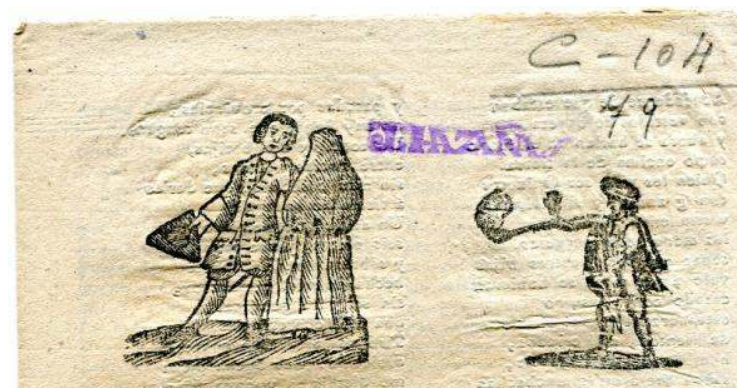

CURIOSO ROMANCE, $Y$ GENERAL BAtalla, que ordinariamente sucede entre los Suegros y Yernos, Suegras y Nueras, cuya comun desdicha es poseida de todos, y deseada de ninguno. Refierese en ella las condiciones, propiedades, y regañoso chasco de la Suegrecil milicia, y la escarmentada Yerneria.

En treamptâa del chasco, que lo Suegrecil sustents. comanmente toesn arma
contra los $Y$ ernos, y Nueras contra los Xernos, y Nuera
Bran clarines los gritos, quanto í tas voces trompetas los ademanes timbores, $y$ las ar was partidideras. Por 12 Vega de Xetafe marchan i to gue tropiezan con andar de hocicos Cuerpo 2 cuergo ta batatts uso frente de las Nueras, los Yernos campo a caupo epusieron fuerza a fuerza y la siguió a toda pricesa el interes, cuya ceño á todo rigor despierta, enpez ciendas de las bodas dicen los suegrositendas; o entendermos lo que perensas Se les dieran nuestras hilis para que fuesen sus negas. Qué es de los dotes y gatas, as bagillas, las haciendas? para que ustedes se verga con ses inanitas lavadas solamente a despender/as? i quieren gastar can damas en juegos, libreas, ferias noramala para ellos, riferimenti alle lamentele dei suoceri che, vedendo come i propri generi dissipavano le fortune avute in dote, si domandavano se «gli si diedero le nostre figlie/ per farle diventare le loro negre?».

Di diverso tenore sono le pubblicazioni ambientate nelle taverne ${ }^{38}$ o riferite al consumo di alcolici. Tra queste troviamo la Relazione burlesca di un ubriaco ${ }^{39}$, dove il protagonista racconta al pubblico quello che era accaduto a lui e al suo compare una volta arrivato a Siviglia, per ottenere dal Reggente il permesso di divorziare da una donna crapulona e sempre incinta. In questo testo si osserva come l'ubriachezza del relatore, oltre a rendere più divertente il racconto, diventi anche una maschera attraverso la quale attaccare la Chiesa, come nei versi «Che al mio funerale/ non

37 CURIOSO ROMANCE, Y GENERAL BA-talla, que ordinariamente sucede entre los Suegros y Yernos, Suegras y Nueras, cuya comun desdicha es poseida de todos, y deseada de ninguno. Refierese en ella las condiciones, propiedades y regañoso chasco de la Suegrecil milicia, y la escarmentada Yernería. Il colofone reca: «Con licencia: En Córdoba, en la Oficina de D. Luis Ramos y Coria, Plazuela de las Cañas, donde se hallará todo género de surtimiento y Estampas». ${ }^{8}$ Nelle Coplas della taverniera e degli ubriachi si descriveva con sarcasmo il microcosmo della taverna, dove la padrona annacqua il vino e gli uomini spendono i loro pochi averi sapendo che a casa saranno picchiati dalle mogli.

39 RELACION BURLESCA DE BORRACHO. Il colofone reca: «Sevilla: Imprenta de la Viuda de Haro». 


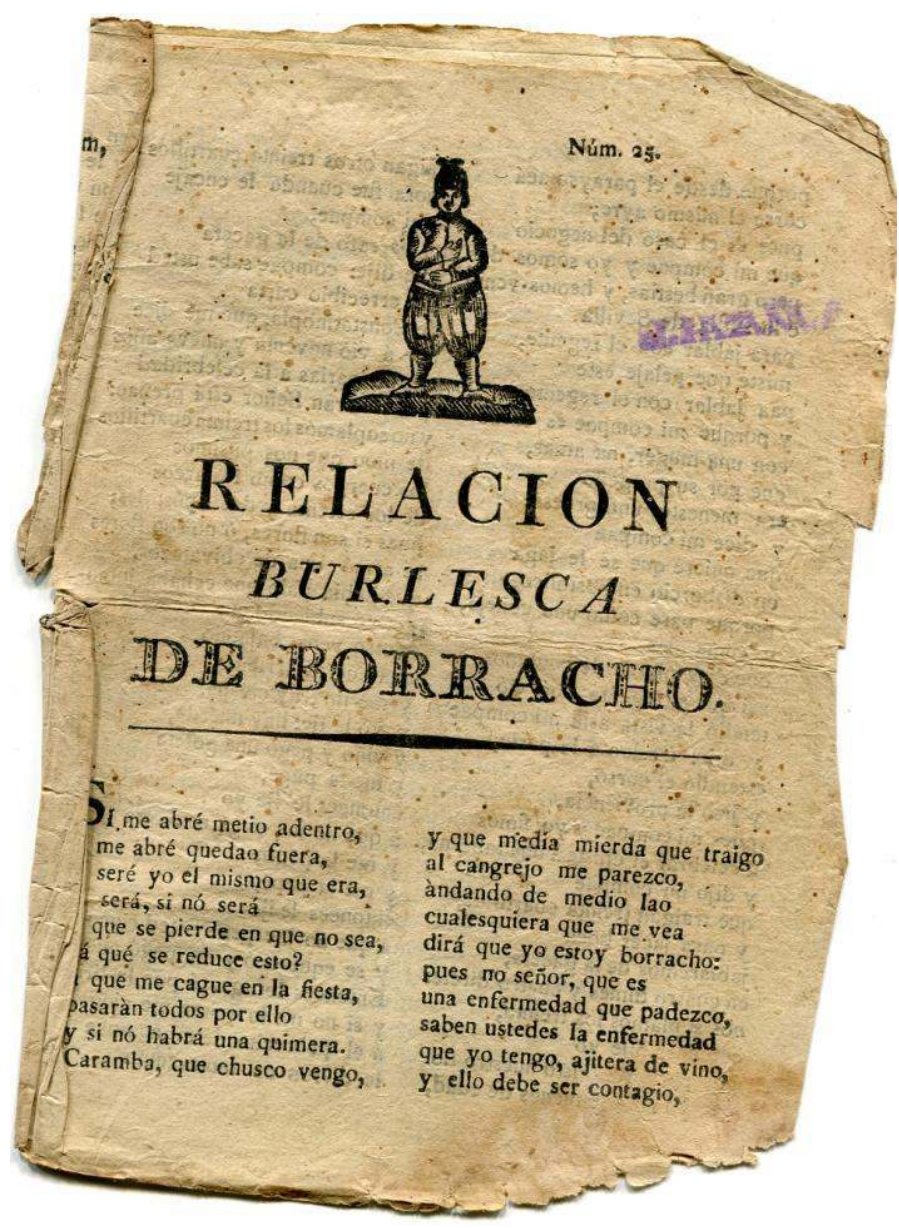

vadano monachelli/ né sacrestani, perché questi/ non fanno niente/ e si portano via quattro reali».

Dello stesso tenore sono i Bandi divertentissimi contro gli ubriachi e le ubriache dove si specificano le multe applicate a qualsiasi specie di ubriachezza ${ }^{40}$ composto da due documenti in prosa. In uno si rendevano pubbliche le decisioni prese dai «signori giudici, né apostolici, né reali, proprietari e fornitori del succo di Noè», nell'altro, firmato da Donna "Ramo d'uva", si dichiarava che le «madamas

caderillas di prima e seconda categoria» avevano deciso di riunirsi in un'altra cantina, «formando e fissando per noialtre, in solidum, le nostre Costituzioni tracannatici».

Era questo mondo carnale, vissuto nelle taverne, ultimi templi urbani consacrati a Bacco, quello in cui passavano il tempo tanto i personaggi di queste storie quanto i lettori-ascoltatori, almeno fino al XX secolo.

Un altro tipo di cordel mescolava storie fantastiche e satira, cercando di far ridere il pubblico dei propri stessi sogni e ambizioni.

Un buon esempio di questa tendenza si trova nella Relazione Burlesca, nella quale è contenuta la scoperta di un’isola chiamata Jauja [...] più ricca e più abbondante di tutto quanto c'è nel mondo, scoperto dallo sfortunato Capitano, chiamato Longares de Semtiom y de Gorgas. Composta da un soldato che viaggiava nel vascello che la scoprì come testimone oculare di tutto quello che qui si riferisce ${ }^{41}$.

40 BANDOS DIVERTIDISIMOS contra los borrachos y borrachas, en que se manifiestan las multas aplicadas à cada especie de borrachera. Il colofone reca: «MADRID: 1846. IMPRENTA DE D. J. M. MARES. Corredera baja de S. Pablo, núm. 27». Di tutti i documenti del corpus solo due, questo e la relazione sul topo delle Canarie che si vedrà più avanti, sono datati.

${ }^{41}$ RELACION BURLESCA, en que se contiene el descubrimiento de una Isla llamada Jauja, [...] mas rica y abundante de todo cuanto hay en el mundo, descubierta por el afortunado Capitan, llamado Longares de Semtiom y de Gorgas. Compuesta por un soldado que iba en el Navio que 


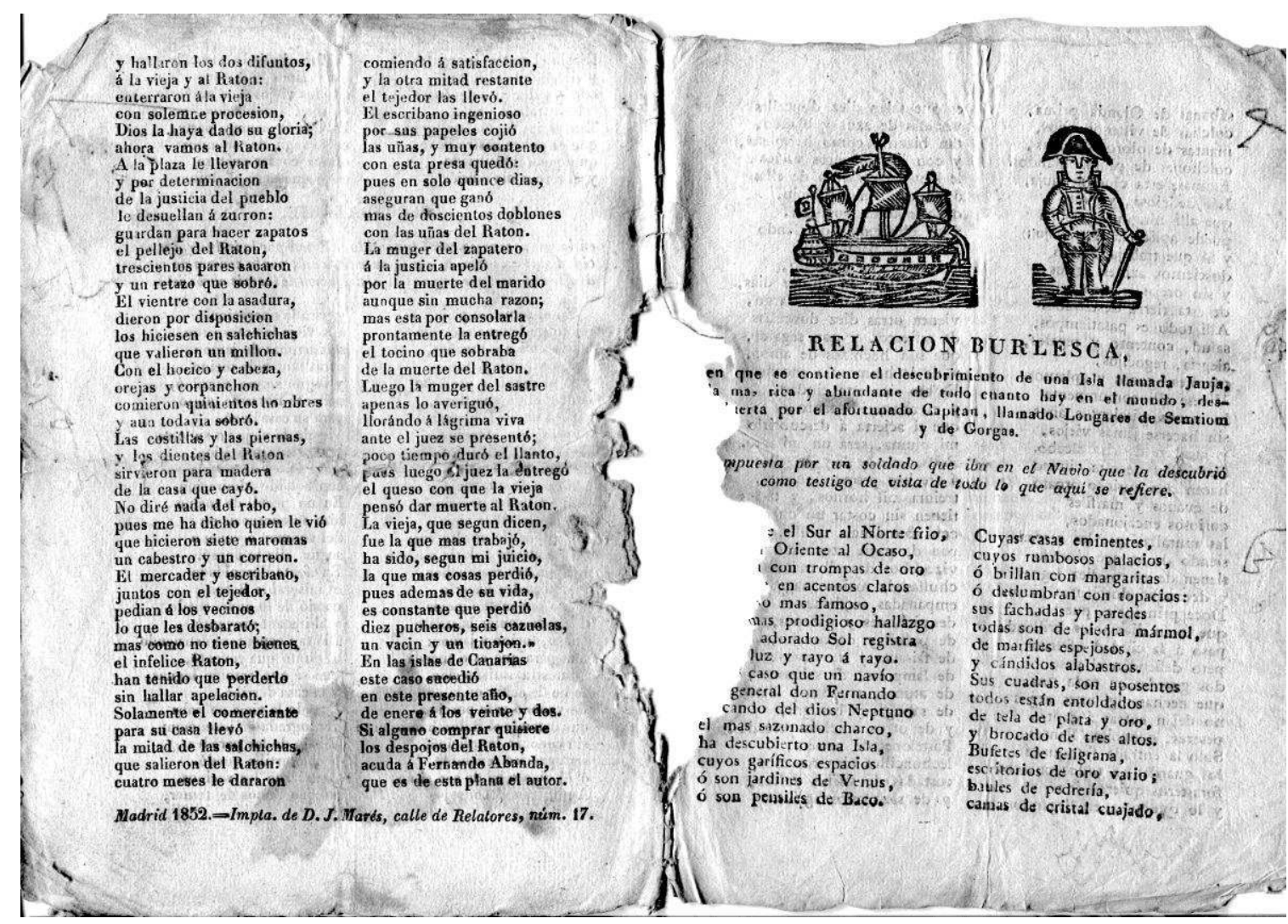

Caro Baroja 42 , dopo aver consultato un'edizione del XVIII secolo, definiva questa relazione come rabelaisiana 43 e la collegava con la letteratura colportage 44 , importata in Spagna, probabilmente da qualche venditore ambulante francese. L'autore descriveva infatti la nuova terra in questi termini esagerati: «Si chiami questa città Jauja/ Isola deliziosa, e tanto/ che li nessuna persona/ può applicarsi al lavoro/ [...] Li tutto è passatempi/ salute, contentezza e regali,/ allegria, gioie,/ piaceri, godimenti e applausi».

Esistevano anche cordel che contenevano una satira di attualità politica, come per esempio Il topo delle Canarie. Relazione graziosa e divertente nella quale si riferiscono le rovine, le morti e gli atti di coraggio eseguite da un topo che si scopri nelle isole delle Canarie, in casa di un tessitore, come consta da una lettera che l'autore ha ricevuto da un suo amico: con gli altri fatti che vedrà il curioso lettore 45.

la descubrió como testigo de vista de todo lo que aquí se refiere. Il colofone reca: « FIN. Valladolid, Imprenta de Santaren».

${ }^{42}$ CARO BAROJA, Julio, op. cit., pp. 209-210.

43 Con questo termine, che deriva dall'autore di Gargantua e Pantagruel, si definisce uno stile caratterizzato da sfrontato umorismo, stravaganti caricature e naturalismo grottesco.

44 Termine francese con cui si definisce la letteratura popolare distribuita dall'altro lato dei Pirenei.

45 EL RATON DE CANARIAS. RELACION graciosa y divertida en que se refieren los estragos, muertes y valentías ejecutadas por un ratón que se descubrió en las islas de Canarias, en casa de un tejedor, segun consta de una carta que recibió el autor, de un amigo suyo: con lo demas 


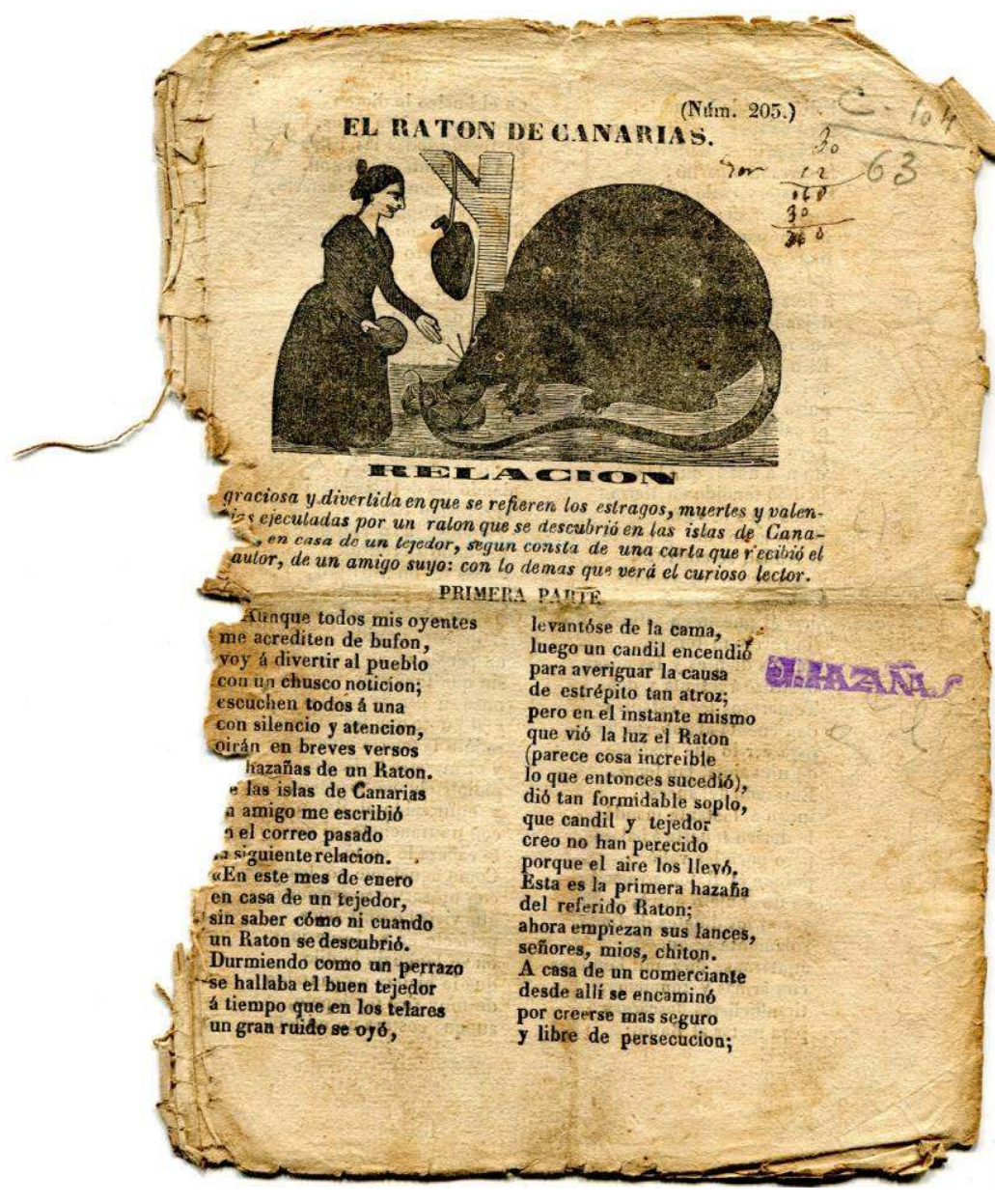

La storia, divisa in due parti, inizia narrando i furti e le scorribande del topo finché gli abitanti, uniti per sconfiggere il nemico comune, provano a ucciderlo con i metodi più esilaranti e disparati, incluso l'impiego dell'esercito, dato che la morte del "topo" avrebbe creato benefici per tutti.

Come ultima categoria resta da analizzare quei romances che Caro Baroja definisce realizzati «ex profeso per essere rappresentati in riunioni» 46 .

In quest'ambito la letteratura di cordel ebbe la sorte di poter contare su un ingegnoso autore, Agustin

Nieto, cui si devono le relazioni burlesche intitolate Il cavallo ${ }^{47}$, La Calle de la Feria e, appunto, La riunione ${ }^{48}$.

que verá el curioso lector. Il colofone reca: «MADRID 1852. Impta. de D. J. M. Marés, calle de Relatores, núm. 17». Stampata nello stesso luogo e simile per impaginazione e carta utilizzata a quella dei Bandi divertentissimi.

${ }^{46}$ CARO BAROJA, Julio, op. cit., p. 211.

47 RELACION BURLESCA INTITULADA DEL CABALLO. Compuesta par Don Augustin Nieto.

48 RELACION BURLESCA INTITULADA: LA TERTULIA. Compuesta por Don Augustin Nieto. Le tre relazioni composte da Agustin Nieto recano il seguente piè di stampa: «Con licencia: En Córdoba, en la Imprenta de Don Rafael García Rodríguez, Calle de la Librería». 

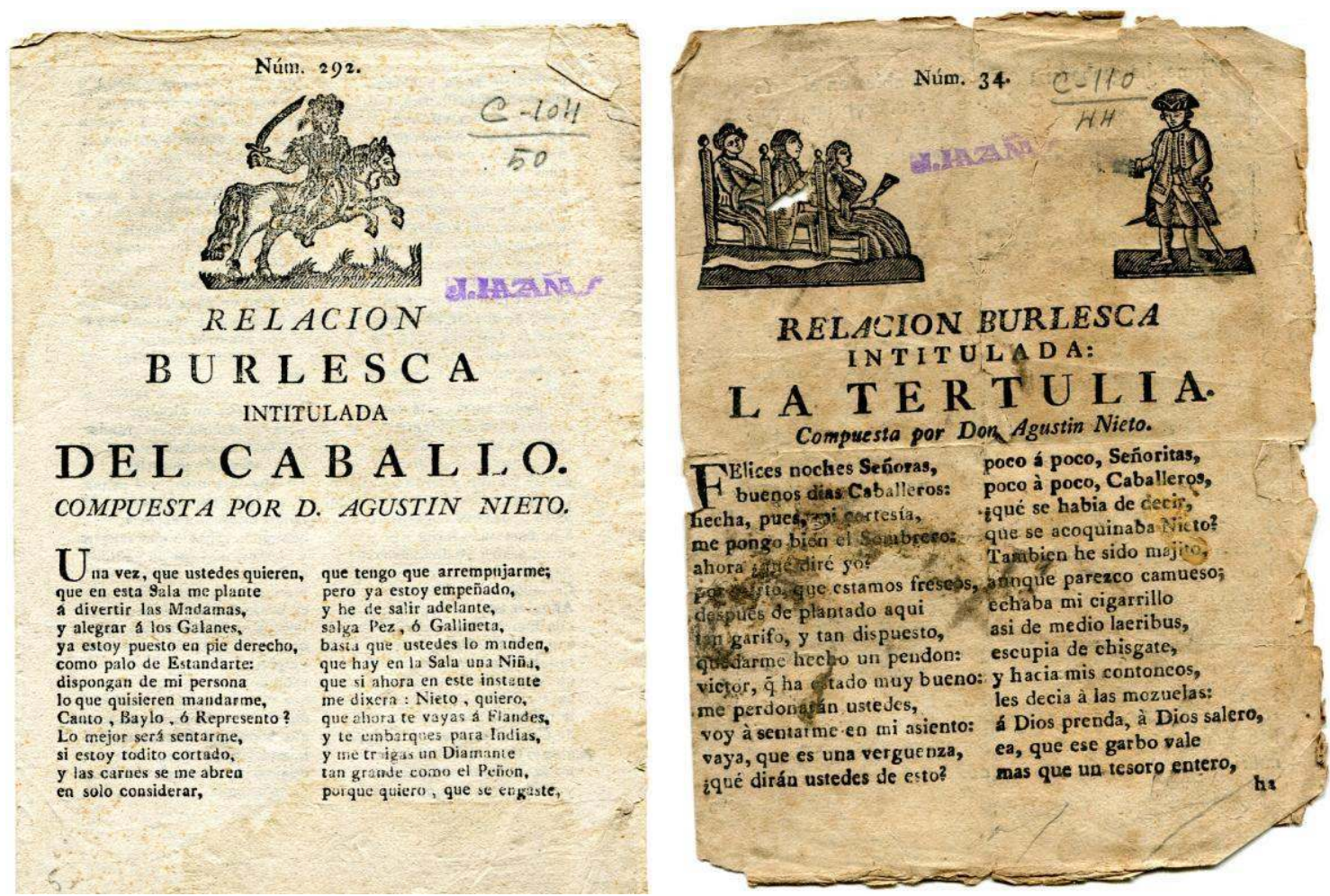

In quest'ultima è presente una descrizione satirica della borghesia Sivigliana e un riferimento al «togliere Giudici, mettere Giudici;/ però tutti, con che accordo/ ognuno mette a suo piacimento/ le cose, e a suo modello!».

La caratteristica saliente di queste opere è l'andalusismo, in altre parole l'impiego del dialetto andaluso, presente soprattutto nelle anomalie ortografiche proprie di chi lo utilizzava come reclame per vendita.

Nella metà del XIX secolo, in un contesto comunicazionale sempre più complesso, questo tipo di letteratura popolare non era l'unico canale di trasmissione dell'umorismo meridionale, visto che doveva convivere con le pubblicazioni satirico politiche liberali nelle quali, soprattutto nei dialoghi, era presente lo stesso uso dell'andaluso.

\section{Conclusioni}

Sebbene si sia diffuso in un'epoca di "manifattura culturale", il cordel può essere definito un prodotto basato sulla mercificazione della cultura e dell'identità popolare $\mathrm{e}$ destinato, soprattutto per il diffuso analfabetismo delle classi subalterne, ad una clientela medio alta.

La specificità di questi stampati sembra avere, come il resto dei generi letterari marginali, la volontà di saziare l'appetito popolare per il violento, il truculento e il passionale, sentimenti esclusi dalla cultura ufficiale. In questo regno delle passioni 
richiesto dal popolo, la satira prendeva forma attraverso l'immagine di "copertina" e l'uso dei topoi e delle storie eccezionali-normali.

La diffusione di alcune pubblicazioni di cordel di tono satirico rappresenta un chiaro indizio di come, nella prima metà dell'Ottocento, queste venissero lette e ascoltate anche come alternativa ai contenuti delle pubblicazioni ufficiali.

Un indizio di quest'antagonismo si può trovare analizzando le misure difensive attuate dal Potere nei confronti del cordel, come la creazione di organismi polizieschi per la vigilanza, con lo scopo di ostacolarne la vendita e la distribuzione.

Quest'ostilità istituzionale sembra confermata dal fatto che la maggior parte dei documenti consultati non reca i dati d'edizione, ma termina semplicemente con la parola «Fine» o l'inscrizione «Proprietà di», mentre la tipologia meno rappresentativa è quella formata dalle pubblicazioni che si concludono con la formula «con licenza», aggiungendo solo il nome dello stampatore, il luogo e la data di stampa.

Questa strategia si potrebbe inserire in un'offensiva più vasta, che controllava la "vera" cultura del popolo, anche attraverso la promozione di periodici satirico-politici, più facili da controllare rispetto ai cordel.

L'analisi dei loro contenuti ci mostra una satira fatta di rime incisive e di velate allusioni che, in epoca di Restaurazione, non esita a evidenziare i vizi del clero, la corruzione politica o demolire il mito dei tabù morali, per cui è opportuno sia osservare più attentamente l'influenza della satira di cordel sullo sviluppo della stampa satirica liberale, sia evidenziarne l'importanza per continuare a scrivere la storia della stampa popolare. 


\section{${ }^{*}$ Los autores}

Francescomaria Evangelisti, $\mathrm{PhD}$ in "Comunicazione e critica della cultura" presso l' Università di Siviglia, è ricercatore in "Storia della propaganda ed analisi della comunicazione politica per COMPOLITICAS". Coordina la ricerca in "Comunicazione e geopolitica nell'ex spazio sovietico" per l'Observatorio Eurasia e collabora con pubblicazioni in lingua italiana e spagnola.

URL: < http://studistorici.com/progett/autori/\#Evangelisti >

Maria Eugenia Gutiérrez Jiménez è laureata in Giornalismo e docente nella facoltà di Comunicazione dell'Universitá di Siviglia. Membro del gruppo di ricerca Storia del giornalismo e Letture popolari in Andalusia. Coordina la sezione "Bibliografica" della pubblicazione I/C. Revista Científica de Información y Comunicación.

URL: < http://studistorici.com/progett/autori/\#GutierrezJimenez >

\section{Per citare questo articolo:}

EVANGELISTI, Francescomaria, GUTIÉRREZ JIMÉNEZ, Maria Eugenia «La satira come prodotto culturale: La letteratura di cordel nella Spagna della prima metà dell'Ottocento», Diacronie. Studi di Storia Contemporanea: La satira fa storia. Eventi, pratiche, linguaggi, 29/10/2012,

URL:< http://www.studistorici.com/2012/10/29/evangelisti-gutierrez-jimenez_numero_11/ >

Diacronie Studi di Storia Contemporanea $\beta$ www.diacronie.it

Risorsa digitale indipendente a carattere storiografico. Uscita trimestrale.

redazione.diacronie@hotmail.it

Comitato di redazione: Marco Abram - Giampaolo Amodei - Jacopo Bassi - Luca Bufarale - Gianluca Canè - Alessandro Cattunar - Alice De Rensis - Barbara Galimberti - Deborah Paci - Fausto Pietrancosta - Matteo Tomasoni - Luca Zuccolo

Diritti: gli articoli di Diacronie. Studi di Storia Contemporanea sono pubblicati sotto licenza Creative Commons 2.5. Possono essere riprodotti a patto di non modificarne i contenuti e di non usarli per fini commerciali. La citazione di estratti è comunque sempre autorizzata, nei limiti previsti dalla legge. 